\title{
Correction to: Detection of Running Malware Before it Becomes Malicious
}

Sergii Banin and Geir Olav Dyrkolbotn

\section{Correction to: \\ Chapter "Detection of Running Malware Before it Becomes Malicious" in: K. Aoki and A. Kanaoka (Eds.): Advances in Information and Computer Security, LNCS 12231, https://doi.org/10.1007/978-3-030-58208-1_4}

Some errors were present in the originally published Chapter 4. The following modifications were made:

Page 67, line 16 has been corrected to: "switching from BEP behavior to AEP it is relatively low".

Page 67, line 22 has been corrected to: "selects more features for AEP data than for BEP data”. 